\title{
Uso de Dalbergia palo-escrito como alternativa vegetal para el curtido de la piel de conejo
}

\section{Use of Dalbergia palo-escrito as a vegetable alternative to rabbit tanned leather}

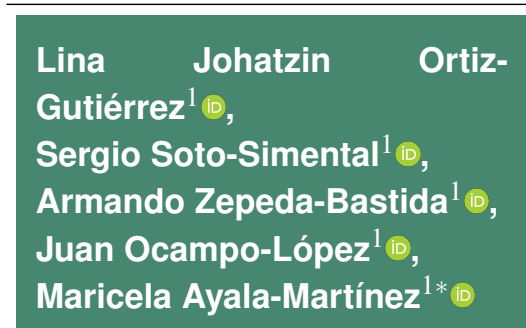

${ }^{1}$ Instituto de Ciencias

Agropecuarias, Universidad

Autónoma del Estado de Hi-

dalgo. Avenida Universidad

$\mathrm{Km} \mathrm{1,} \mathrm{s/n,} \mathrm{Ex-Hacienda} \mathrm{de}$ Aquetzalpa, CP. 43600 . Tulancingo de Bravo, Hidalgo, México.

*Autor de correspondencia: ayalam@uaeh.edu.mx

Nota científica

Recibida: 05 de marzo 2021

Aceptada: 18 de septiembre 2021

Como citar: Ortiz-Gutiérrez LJ, Soto-Simental S, ZepedaBastida A, Ocampo-López J, Ayala-Martínez M (2021) Uso de Dalbergia palo-escrito como alternativa vegetal para el curtido de la piel de conejo. Ecosistemas y Recursos Agropecuarios 8(3): e2967. DOI: 10.19136/era.a8n3.2967
RESUMEN. Se evaluó el uso de Dalbergia Palo-escrito, como curtiente natural de piel de conejo. Se realizaron dos ensayos con extractos acuosos de Dalbergia palo-escrito, para el primero se utilizó hoja y tallo, para el segundo solo se utilizó tallo a concentración de 20,40 y $60 \mathrm{~g} \mathrm{~L}^{-1}$, se realizó el curtido de piel de conejo y se evaluó color, textura y propiedades físicas de la piel. Al utilizar extracto de hoja y $60 \mathrm{~g} \mathrm{~L}^{-1}$ de tallo disminuyó la luminosidad $(P<0.05)$. Las pérdidas de peso de la piel, firmeza $y$ fracturabilidad se incrementaron con $40 \mathrm{~g} \mathrm{~L}^{-1}$; y con 20,40 y $60 \mathrm{~g} \mathrm{~L}^{-1}$ las pérdidas de área y la proporción de tamaño disminuyeron, e incrementó suavidad, brillo y firmeza. Las pieles de conejo curtidas con extractos de Dalbergia palo-escrito a concentración de $40 \mathrm{~g} \mathrm{~L}^{-1}$, presentaron mejor calidad física.

Palabras clave: Cuero, curtiente, hojas, tallo, taninos.

ABSTRACT. Evaluate the Dalbergia palo-escrito, as a natural tanning agent for rabbit skin. Two tests were carried out with extracts of $D$. paloescrito, for the first, with leaf and stem, for the second the stem at different concentrations $\left(20,40\right.$ and $\left.60 \mathrm{~g} \mathrm{~L}^{-1}\right)$. With the extract, it carried out the tanning of rabbit skin, to evaluate color, texture and physical properties of the skin. The leaf and $60 \mathrm{~g} \mathrm{~L}^{-1}$ of stem extract decreased $(P<0.05)$ luminosity, while it did not affect the loss of weight and size of the skin. The loss of skin weight, firmness and fracturing increased with $40 \mathrm{~g} \mathrm{~L}^{-1}$; with 20,40 and $60 \mathrm{~g} \mathrm{~L}^{-1}$ the loss of area and the proportion of size decreased, instead they increased softness, gloss and firmness. Concluded that the rabbit skins tanned with extracts of $D$. palo-escrito at a concentration of $40 \mathrm{~g} \mathrm{~L}^{-1}$, presented better physical quality.

Key words: Leather, tanning agent, leaves, stem, tannins. 


\section{INTRODUCCIÓN}

Durante el proceso de curtido se estabiliza el colágeno de la piel mediante la formación de puentes de hidrógeno entre el curtiente y los aminoácidos de la proteína, lo que proporciona resistencia a los microorganismos y características hidrotérmicas (Shegaw et al. 2020), por lo que, la piel putrescible se convierte en cuero imputrescible (Pinto 2013). El curtido vegetal, es el método más antiguo, utiliza compuestos como taninos, metabolitos secundarios solubles en agua, con capacidad de precipitar la proteína (colágeno o corium) y alcaloides, para producir estabilidad hidrotérmica (Shegaw et al. 2020), se encuentran en cortezas (Uzma et al. 2020), maderas, frutos, hojas o vainas de diversas especies vegetales (López 1986).

La industria del curtido está buscando alternativas que permitan reemplazar la utilización de cromo por otros curtientes, por ello se han propuesto el uso de extractos obtenidos de hojas, semillas, tallos o cortezas de una gran diversidad de plantas como las Acacias (Maina et al. 2019, China et al. 2021). Un árbol endémico de la Sierra Madre Oriental de México, de la familia Fabaceae es Dalbergia palo-escrito, el cual debido a la belleza, durabilidad y excelentes propiedades físicas, mecánicas y acústicas de su madera tiene un alto valor económico e importancia comercial internacional; pero una de sus características importantes es la cantidad de taninos que contiene la hoja y tallo, siendo las hojas desechadas debido a que no tienen importancia económica (Rzedowski y Guridi 1988). Por lo anterior, el objetivo del presente trabajo fue evaluar un extracto acuoso de $D$. palo-escrito en el proceso del curtido de piel de conejo.

\section{MATERIALES Y MÉTODOS}

La planta de Dalbergia palo-escrito se obtuvo del invernadero del Instituto de Ciencias Agropecuarias de la Universidad Autónoma del Estado de Hidalgo (ICAp-UAEH) localizado a $20^{\circ} 03^{\prime}$ 45.4" LN y $98^{\circ} 22$ ' 52.9" LO, las hojas y tallos se deshidrataron a $25{ }^{\circ} \mathrm{C}$, bajo la sombra, para luego molerlos y conservarlos en bolsas herméticas a la misma temperatura. Para el primer experimento, se obtuvieron extractos acuosos a partir de hoja y tallo de $D$. palo-escrito de forma separada a concentración de $80 \mathrm{~g} \mathrm{~L}^{-1}$, equivalentes al $12 \%$ de ácido tánico. En el segundo experimento se obtuvieron extractos acuosos de tallos de $D$. palo-escrito a concentración de 20, 40 y $60 \mathrm{~g} \mathrm{~L}^{-1}$. Para obtener los extractos acuosos de $D$. palo-escrito, el material vegetal, deshidratado y molido, se disolvió en $1 \mathrm{~L}$ de agua y se mantuvo en ebullición durante 30 minutos; posteriormente la solución obtenida, se filtró a través de tres capas de gasa, para obtener la parte líquida, la cual se conservó en congelación a $-20{ }^{\circ} \mathrm{C}$.

Las pieles empleadas, se obtuvieron de conejos de 63 días de edad, de la raza California y Mariposa, los animales se sacrificaron de acuerdo con la NOM-033-SAG-ZOO-2014 (SAGARPA 2015). Para obtener la piel, se realizó un corte de las patas anteriores a la altura de los carpos, posteriormente se realizó un corte circular en los tarsos, para luego proceder al desollado, jalando la piel hacia abajo, la piel se retiró estando la canal caliente, para luego despojar de los restos de tejido y grasa hasta tener la piel limpia y luego conservar en congelación a $-20{ }^{\circ} \mathrm{C}$, hasta su utilización.

En el primer experimento, se utilizaron 12 pieles divididas a la mitad y asignadas completamente al azar a cuatro tratamientos (cromo, ácido tánico, extracto de hoja y extracto de tallo), para el segundo experimento, se utilizaron 42 pieles, las cuales se dividieron a la mitad, y en cada mitad de ellas se aplicaron los siguientes tratamientos: ácido tánico sintético al $12 \%$, extracto de $D$. palo-escrito 20 , extracto de $D$. palo-escrito 40 y extracto de $D$. paloescrito $60 \mathrm{~g} \mathrm{~L}^{-1}$.

El curtido de las pieles, se realizó de acuerdo a las recomendaciones de Alfonso (2015), descongelandolas a $4{ }^{\circ} \mathrm{C}$, para después hidratarlas y lavarlas con $20 \mathrm{~g}$ de detergente biodegradable y $20 \mathrm{~g}$ de $\mathrm{NaHCO}_{3}$, en $2 \mathrm{~L}$ de agua, para después dejarlas reposar durante $24 \mathrm{~h}$, y enjuagarlas con agua corriente, hasta eliminar los residuos de detergente. Las pieles se dividieron de acuerdo al tratamiento correspondientes y se sometieron a un pre-curtido 
con $27 \mathrm{~g}$ de $\mathrm{NaCl}, 27 \mathrm{~g} \mathrm{Al}_{2}\left(\mathrm{SO}_{4}\right)_{3}$ y $27 \mathrm{~g}$ de $\mathrm{K}_{2} \mathrm{SO}_{4}$ $\mathrm{L}^{-1}$, para después dejarlas reposar por $24 \mathrm{~h}$ y escurrir sin enjuagar durante $2 \mathrm{~h}$. Posteriormente se procedió al curtido de acuerdo al tratamiento asignado (Tabla 1), las pieles se dejaron en la solución curtiente durante $72 \mathrm{~h}$, con agitación cada $12 \mathrm{~h}$, una vez transcurrido el tiempo se enjuagaron y dejaron escurrir por $2 \mathrm{~h}$. Posteriormente, se sumergieron en solución de $\mathrm{NaHCO}_{3}\left(10 \mathrm{~g} \mathrm{~L}^{-1}\right)$, con agitación constante durante 30 minutos, para posteriormente enjuagarlas y secarlas a la sombra por $72 \mathrm{~h}$. Las pieles secas, se aceitaron con $5 \mathrm{~mL}$ de una mezcla de $70 \mathrm{~mL}$ de aceite y $30 \mathrm{~mL}$ de agua, posteriormente se aflojaron con movimientos de tracción para hacerlas flexibles. Para el acabado, las pieles se friccionaron con una mezcla de aserrín y gasolina blanca, con la finalidad de eliminar el pelo que no se fijó durante el curtido, para luego lijar y uniformizar la superficie sin pelo. La cantidad de taninos de $D$. palo-escrito, se determinó por medio del método cuantitativo con el reactivo Folin-Denis como lo describe Gutiérrez et al. (2000), una vez realizada la reacción se midió a una absorbancia de $760 \mathrm{~nm}$ en un espectrofotómetro Jenway modelo 6305 (Dunmow Esse, UK).

Para la determinación de color, se utilizó la escala de colores CIEL*a*b*, para ello se empleó un colorímetro Minolta modelo CM-508d (Tokio, Japón), configurando el iluminante D65 y observador de $10^{\circ}$, para obtenerlos valores de luminosidad $\left(L^{*}\right)$, escala de rojos $\left(a^{*}\right)$ y la escala de amarillos $\left(b^{*}\right)$ (AMSA 2012). La prueba de penetración de la piel se realizó con un texturómetro Brookfield CT3 (Brookfield, Middleboro, MA, USA), acoplado a una sonda en forma de aguja de $0.6 \mathrm{~mm}$ de diámetro, con velocidad de la prueba de $1 \mathrm{~mm} \mathrm{~s}^{-1}$, para obtener los valores de dureza, trabajo de dureza y fracturabilidad de la piel, con el software Texture Pro CT versión 1.4 (Brookfield, Middleboro, MA, USA).

Se determinó la pérdida de peso de las pieles, con una balanza Scout Pro modelo SP402 (Ohaus, Pine Brook, NJ, USA) pesando la piel antes y después del proceso de curtido. Posteriormente, con la finalidad de obtener la pérdida de área de la piel, se midió la superficie de la piel con una regla antes y después del proceso de curtido. También se de- terminó la perdida de tamaño, para lo cual se colocaron ( $5 \times 5 \mathrm{~cm}$ de piel) en un vaso de precipitado con agua en ebullición por 5 minutos, posteriormente se retiraron y dejaron secar, para volver a medirlas, de esta manera obtener la diferencia de áreas, antes y después de hervir.

Los datos obtenidos se analizaron bajo un análisis de varianza completamente al azar, para determinar diferencias entre tratamientos se realizó una prueba de comparación de medias de Tukey $(\mathrm{P}>$ 0.05). Todos los análisis se realizaron utilizando el software SPSS ver 20.

\section{RESULTADOS Y DISCUSIÓN}

Aunque a nivel mundial, el proceso de curtido se basa en el tanino de cromo, como producto principal en un $80 \%$ de la industria del cuero, por su eficiencia y versatilidad; actualmente esta industria está exigiendo remplazar el cromo, por taninos obtenidos de extractos de hojas, semillas, tallos y corteza de plantas (Correa et al. 2019). Los extractos acuosos de Dalbergia palo-escrito, tuvieron una concentración de taninos (ácido tánico) de 1.31 con hoja y 1.20\% con tallo, respectivamente, los cuales son superiores a los reportados por Cabrera-Carrión et al. (2017) en extractos acuosos de hoja de $M$. olerifera $(0.71$ a $1.06 \%)$ de 12, 15 y 18 meses de edad; y menores a lo obtenido en Oreganum vulgare (orégano) (8\%), Potentilla erecta (18.1\%) (Maier et al. 2017), así como con extractos de corteza de Pinus pinaster, los cuales encontraron valores entre 30 y $60 \%$ (Seabra et al. 2017).

El color de las pieles curtidas con ácido tánico sintético, extracto de hoja y de tallo, no fue diferente estadísticamente para el parámetro $L^{*}$ (Tabla 2), pero tuvo valores menores que el tratamiento con cromo; en cambio el parámetro $a^{*}$ fue menor en las pieles curtidas con cromo y ácido tánico sintético. Para el parámetro $b^{*}$, no se detectaron diferencias estadísticas $(P>0.05)$ en las pieles curtidas con cromo, así como, con los extractos de hoja y tallo, con valores mayores a las del tratamiento con ácido tánico sintético, lo cual indica que es factible utilizar extractos de $D$. palo-escrito, ya que se mantiene el color, debido 
Tabla 1. Reactivos utilizados en la solución curtiente de pieles de conejo, para los diferentes experimentos.

\begin{tabular}{lcccccccc}
\hline \multirow{2}{*}{ Reactivo } & \multicolumn{3}{c}{ Experimento $1^{1}$} & \multicolumn{5}{c}{ Experimento $2^{2}$} \\
& Cromo & Ácido & Hoja & Tallo & Ácido & 20 & 40 & 60 \\
& & tánico & & & tánico & & $\mathrm{g} \mathrm{L}^{-1}$ & \\
\hline $\mathrm{NaCl} \mathrm{g})$ & 27 & 27 & 27 & 27 & 27 & 27 & 27 & 27 \\
$\left(\mathrm{NH}_{4}\right)_{2} \mathrm{SO}_{4}(\mathrm{~g})$ & 27 & 27 & 27 & 27 & 27 & 27 & 27 & 27 \\
$\mathrm{Al}_{2}\left(\mathrm{SO}_{4}\right)_{3}(\mathrm{~g})$ & 27 & 27 & 27 & 27 & 27 & 27 & 27 & 27 \\
$\mathrm{CH}_{3} \mathrm{COOH}(\mathrm{mL})$ & 3 & 3 & 3 & 3 & 3 & 3 & 3 & 3 \\
$\mathrm{C}_{76} \mathrm{H}_{52} \mathrm{O}_{46}(\mathrm{~g})$ & 0 & 12 & 0 & 0 & 12 & 0 & 0 & 0 \\
$\mathrm{Cr}_{2}\left(\mathrm{SO}_{4}\right)_{3} 12 \mathrm{H}_{2} \mathrm{O}(\mathrm{g})$ & 27 & 0 & 0 & 0 & 0 & 0 & 0 & 0 \\
Extracto de hoja $(\mathrm{mL})^{*}$ & 0 & 0 & 550 & 0 & 0 & 0 & 0 & 0 \\
Extracto de tallo $(\mathrm{mL})^{* *}$ & 0 & 0 & 0 & 680 & 0 & 900 & 850 & 870
\end{tabular}

${ }^{1}$ Los tratamientos son cromo $(27 \mathrm{~g})$, ácido tánico sintético (12\%), extracto de hoja Dalbergia paloescrito ( $80 \mathrm{~g} \mathrm{~L}^{-1}$, equivalente al $12 \%$ ácido tánico) y extracto de tallo de Dalbergia palo-escrito ( 80 $\mathrm{g} \mathrm{L}^{-1}$, equivalente al $12 \%$ ácido tánico) *, 2 Los tratamientos son ácido tánico $12 \%, 20,40$ y $60 \mathrm{~g}$ $\mathrm{L}^{-1}$ de extractos de tallo de Dalbergia palo-escrito**

Tabla 2. Calidad de piel de conejo, curtida con extractos acuosos de hoja y tallo de Dalbergia paloescrito.

\begin{tabular}{lcccc}
\hline & Cromo $^{1}$ & Ácido tánico $^{2}$ & Hoja $^{3}$ & Tallo $^{3}$ \\
\hline $\mathrm{L}^{*}$ & $69.36 \pm 4.29^{a}$ & $62.61 \pm 7.42^{b}$ & $56.42 \pm 5.69^{c}$ & $61.94 \pm 4.93^{b}$ \\
$\mathrm{a}^{*}$ & $-0.53 \pm 1.63^{b}$ & $-5.32 \pm 7.11^{c}$ & $3.38 \pm 2.07^{a}$ & $4.47 \pm 1.13^{a}$ \\
$\mathrm{~b}^{*}$ & $22.03 \pm 2.04^{a}$ & $4.56 \pm 0.79^{b}$ & $20.70 \pm 2.81^{a}$ & $20.07 \pm 1.10^{a}$ \\
Pérdida de peso $(\mathrm{g})$ & $82.40 \pm 10.24$ & $85.07 \pm 2.72$ & $88.35 \pm 6.24$ & $88.85 \pm 4.54$ \\
Pérdida de tamaño (cm) & $17.31 \pm 5.02$ & $18.69 \pm 4.91$ & $12.04 \pm 8.79$ & $16.55 \pm 6.28$ \\
Pérdida de área $\left(\mathrm{cm}^{2}\right)$ & $57.05 \pm 5.45^{a}$ & $18.69 \pm 4.91^{b}$ & $47.67 \pm 14.38^{a}$ & $44.31 \pm 15.14^{a}$ \\
Firmeza & $6.53 \pm 2.14^{a}$ & $5.04 \pm 1.01^{b}$ & $4.17 \pm 1.42^{b}$ & $5.17 \pm 1.95^{b}$ \\
Fracturabilidad & $4.45 \pm 3.70^{a b}$ & $4.45 \pm 3.70^{a b}$ & $2.68 \pm 2.05^{c}$ & $5.17 \pm 1.95^{a}$ \\
\hline
\end{tabular}

Media \pm Desviación estándar. ${ }^{a b c}$ Literales diferentes entre columnas indican diferencia estadísticamente significativa $(P<0.05)$. $L^{*}$ : Luminosidad, $a^{*}$ : Valores de rojo a verde, $b^{*}$ : Valores de azul a amarillo, ${ }^{1} \mathrm{Cr}_{2}\left(\mathrm{SO}_{4}\right)_{3} 12 \mathrm{H}_{2} \mathrm{O}(27 \mathrm{~g}),{ }^{2}$ Ácido tánico sintético $(12 \%),{ }^{3}$ Extracto de hoja y extracto de tallo de Dalbergia palo-escrito ( $80 \mathrm{~g} \mathrm{~L}^{-1}$, equivalente al $12 \%$ ácido tánico).

a que los cambios de color en la luminosidad, como en las escalas de rojos y amarillos esta influenciadas por los agentes de curado, taninos o cromo, lo que coincide con Berhanu y Ratnapandian (2017), quienes optimizaron diversos parámetros en el curtido de piel de borrego al utilizar corteza de Cassia singueana Del. (mukengeta), mango (Mangifera indica L) y hojas de sábila (Aloe vera), con colores menos oscuros con tendencia a colores rojos $\left(a^{*}\right)$ y prevaleciendo el color de los amarillos $\left(b^{*}\right)$. La pérdida de peso y de tamaño, no fue diferente estadísticamente $(P>0.05)$, entre los tratamientos utilizados para curtir las pieles, en cambio la pérdida de área fue menor al utilizar ácido tánico sintético, mientras que para los extractos de hoja y tallo no fueron diferentes al tratamiento con cromo. En tanto que la firmeza y la fracturabilidad fueron menores con el extracto de hoja de $D$. palo-escrito $(\mathrm{P}<0.05)$, lo que coincide con lo reportado por Shegaw et al. (2020), quienes al incluir
$20 \%$ de extracto de Rumex abyssinicus, obtuvieron pieles más suaves.

En el segundo experimento al utilizar como curtiente extracto acuoso de tallo de $D$. palo-escrito en diferentes concentraciones $\left(20,40\right.$ y $\left.60 \mathrm{~g} \mathrm{~L}^{-1}\right)$ (Tabla 3 ), disminuyó la luminosidad $\left(\mathrm{L}^{*}\right)$, con el incremento de la concentración del extracto, contrario a los colores rojos $\left(\mathrm{a}^{*}\right)$, los cuales no se vieron afectados por la concentración. Sin embargo, los valores fueron mayores a lo obtenido con ácido tánico sintético; en los colores azules-amarillos $\left(b^{*}\right)$, los extractos de tallo de $D$. palo-escrito, presentaron valores menores a los obtenidos con ácido tánico sintético, lo que coincide con lo encontrado por Kusumawati et al. (2016), quienes realizaron un estudio utilizando índigo (Indigofera tinctoria L.) como colorante natural en la piel de pescado con concentraciones del extracto de 20, 25 y $30 \%$, obteniendo tonalidades azules, lo que se puede deber a que esta planta tiene 
Tabla 3. Calidad de piel curtida con ácido tánico y diferentes concentraciones de tallo de Dalbergia palo-escrito.

\begin{tabular}{|c|c|c|c|c|}
\hline & Ácido tánico ${ }^{1}$ & $20^{2}$ & $\begin{array}{l}40^{2} \\
\mathrm{~g} \mathrm{~L}^{-1} \\
\end{array}$ & $60^{2}$ \\
\hline $\mathrm{L}^{*}$ & $74.33 \pm 3.91^{a}$ & $67.80 \pm 5.88^{b}$ & $63.61 \pm 6.07^{c}$ & $59.80 \pm 6.20^{d}$ \\
\hline$a^{*}$ & $-2.11 \pm 0.85^{c}$ & $5.86 \pm 6.03^{a}$ & $3.44 \pm 1.45^{b}$ & $4.64 \pm 1.23^{a b}$ \\
\hline$b^{*}$ & $22.67 \pm 2.21^{a}$ & $18.11 \pm 2.22^{b}$ & $18.31 \pm 2.16^{b}$ & $18.59 \pm 1.50^{b}$ \\
\hline Pérdida de peso(g) & $70.63 \pm 6.72$ & $76.19 \pm 3.93$ & $79.45 \pm 5.03$ & $72.43 \pm 12.29$ \\
\hline Pérdida de tamaño $(\mathrm{cm})$ & $14.66 \pm 12.4^{a b}$ & $33.51 \pm 20.8^{a}$ & $9.98 \pm 18.56^{b}$ & $15.53 \pm 10.56^{a b}$ \\
\hline Pérdida de área $\left(\mathrm{cm}^{2}\right)$ & $67.73 \pm 12.94$ & $59.67 \pm 10.63$ & $56.31 \pm 8.92$ & $57.09 \pm 12.96$ \\
\hline Firmeza & $6.04 \pm 2.45^{a b}$ & $4.43 \pm 2.39^{c}$ & $7.27 \pm 2.69^{a}$ & $4.84 \pm 1.53 \mathrm{bc}$ \\
\hline Trabajo de dureza & $0.02 \pm 0.01^{b}$ & $0.02 \pm 0.01^{b}$ & $0.04 \pm 0.02^{a}$ & $0.02 \pm 0.01^{b}$ \\
\hline Fracturabilidad & $4.95 \pm 3.29^{a b}$ & $3.14 \pm 2.51^{b}$ & $5.76 \pm 3.86^{a}$ & $3.80 \pm 2.34^{a b}$ \\
\hline
\end{tabular}

un contenido de $0.08 \%$ de taninos. Por lo que se puede inferir que, es factible utilizar extracto de tallo y hoja de $D$. palo-escrito, en el proceso de curtido, ya que contienen 1.2 y $1.4 \%$ de taninos en forma de ácido tánico, respectivamente; considerando siempre que las características de las pieles son influenciadas por la cantidad de material vegetal utilizado. La pérdida de tamaño fue mayor con extracto de tallo de D. palo-escrito con 20 y $40 \mathrm{mg} \mathrm{L}^{-1}$, sin presentar diferencia con el tratamiento en el que se utilizó ácido tánico sintético, la firmeza, dureza y fracturabilidad, fue mayor al incluir $40 \mathrm{mg} \mathrm{L}^{-1}$ de extracto de $D$. paloescrito, lo cual indica resistencia en la piel, como lo demostraron China et al. (2020), quienes indican que la concentración de extractos de taninos a partir de diversas especies de Acacias, tienen efecto sobre las propiedades mecánicas de las pieles curtidas. Sobre lo mismo, Guo et al. (2020) encontraron que taninos extraídos a partir de corteza de Coriaria nepalensis, penetra entre las fibras de colágeno de la piel, lo que hace que la superficie sea firme y delicada. Por lo que, la resistencia mecánica y fuerza de rompimiento de la piel curtida, está relacionada con el grosor y heterogeneidad que esta tenga. Al respecto Sundar y Muralidharan (2020) mencionan que los taninos, son adecuados para transformar el colágeno en cuero. Por lo que las pieles curtidas con extractos acuosos de hojas y tallos de Dalbergia palo-escrito, presentaron mejor calidad que con ácido tánico sintético y sulfato de cromo, por lo que es factible de utilizarse como curtientes vegetales.

\section{AGRADECIMIENTOS}

Al Dr. Alfonso Suárez Islas, por proporcionar el material vegetal y hacer su identificación.

\section{LITERATURA CITADA}

Alfonso CC (2015) Biocueros: cueros de pieles marinas curtidas con savia de la musa paradisiaca. Revista de Iniciación Científica 1: 46-50.

AMSA (2012) Meat color measurement guide lines. American Meat Science Association. Champaign. II. USA. https://meatscience.org/publications-resources/printed-publications/amsa-meat-color-measurement-guideli nes. Fecha de consulta: 31 de Agosto de 2021

Berhanu T, Ratnapandian S. (2017) Extraction and Optimization of Natural Dye from Hambo Hambo (Cassia singueana) Plant Used for Coloration of Tanned Leather Materials. Advances in Materials Science and Engineering 1-6. https://doi.org/10.1155/2017/7516409

Cabrera-Carrión JL, Jaramillo-Jaramillo C, Dután-Torres F, Cun-Carrión J, García PA, Rojas de Astudillo L (2017) 
Variación del contenido de alcaloides, fenoles, flavonoides y taninos en Moringa oleifera Lam. en función de su edad y altura. Bioagro 29: 52-60.

Correa RDE, Romero GBM, León RNoemí (2019) Extracción de taninos de semilla de mango criollo (Mangifera indica L.) y su aplicación como curtiente. Journal of Agro-industry Sciences 1: 51-55.

China CR, Nyandoro SS, Munissi JJE, Maguta MM, Meyer M, Schroepfer M (2021) Tanning capacity of Tessmannia burttii extracts: the potential eco-friendly tanning agents for the leather industry. Journal of Leather Science and Engineering 3: 13. DOI: 10.1186/s42825-021-00055-2.

Guo L, Qiang TMY, Wang K, Du K (2020) Optimization of tannin extraction from Coriaria nepalensis bark as a renewable resource for use in tanning. Industrial Crops and Products 149: 112360. DOI: 10.1016/j.indcrop.20 20.112360 .

Gutiérrez GYI, Miranda MM, Varona TN (2000) Validación de 2 métodos espectrofotométricos para la cuantificación de taninos y flavonoides (quercetina) en Psidium guajaba L. Revista Cubana Farmacología 34: 50-55.

Kusumawati F, Riyadi PH, Rianingsih L (2016) Applications indigo (Indigofera Tinctoria L.) as natural dyeing in milkfish [Chanos Chanos (Forsskal, 1775)] skin tanning process. Aquatic Procedia 7: 92-99.

López LH (1986) Manual de métodos de análisis para el químico curtidor. CIATEG. León, Guanajuato. 671p.

Maier M, Oelbermann AL, Renner M, Weidner E (2017) Screening of European medicinal herbs on their tannin content - New potential tanning agents for the leather industry. Industrial Crops and Products 99: 19-26.

Maina P, Moses AO, Nthiga EW (2019) Trends in leather processing: A review. International Journal of Scientific and Research Publications 9: 212-223.

SAGARPA (2015) NOM-033-SAG/ZOO Norma Oficial Mexicana, Métodos para dar muerte a los animales domésticos y silvestres. http://www.dof.gob.mx/nota_detalle.php?codigo=5405210\&fecha=26/08/2015. Fecha de consulta: 31 de agosto de 2021.

Pinto J (2013) Materiales para fabricación o reparación de artículos y complementos de guarnicionería. IC Editorial. Málaga, España. 294p.

Rzedowski J, Guridi G, Lydia I (1988) El palo escrito, un árbol de madera preciosa una nueva especie mexicana de Dalbergia (Leguminosae, Papilionoideae). Acta Botánica Mexicana 4: 1-8.

Seabra IJ, Chim RB, Salgueiro P, Braga ME, De Sousa HC (2017) Influence of solvent additives on the aqueous extraction of tannins from pine bark: potential extracts for leather tanning. Journal of Chemical Technology \& Biotechnology 93: 1169-1182.

Shegaw AM, Ariram N, Balaraman M, Berhanu AD (2020) Rumex abyssinicus (mekmeko): A newer alternative for leather manufacture. Environmental progress and sustainable energy 39: e13453. DOI: 10.1002/ep.13453.

Sundar VJ, Muralidharan C (2020) Environmentally friendly mineral-free tanning of animal skins-sustainable approach with plant resources. Environmental Processes 7: 255-270.

Uzma A, Tahira AM, Zeb S, Muhammad AA (2020) Vegetable tanning of sole fish skin by using tannins extracted from plants. Asian Journal of Research in Biosciences 2: 1-9. 